\title{
Breakdown of the Two-Step Model in $K$-Shell Photoemission and Subsequent Decay Probed by the Molecular-Frame Photoelectron Angular Distributions of $\mathrm{CO}_{2}$
}

\author{
X.-J. Liu, ${ }^{1}$ H. Fukuzawa, ${ }^{1}$ T. Teranishi, ${ }^{1}$ A. De Fanis, ${ }^{1}$ M. Takahashi,${ }^{1}$ H. Yoshida, ${ }^{2}$ A. Cassimi, ${ }^{3}$ A. Czasch, ${ }^{4}$ L. Schmidt, ${ }^{4}$ \\ R. Dörner, ${ }^{4}$ K. Wang, ${ }^{5}$ B. Zimmermann, ${ }^{5}$ V. McKoy, ${ }^{5}$ I. Koyano, ${ }^{6}$ N. Saito, ${ }^{7}$ and K. Ueda ${ }^{1, *}$ \\ ${ }^{1}$ Institute of Multidisciplinary Research for Advanced Materials, Tohoku University, Sendai 980-8577, Japan \\ ${ }^{2}$ Department of Physical Science, Hiroshima University, Higashi-Hiroshima 739-8526, Japan \\ ${ }^{3}$ CIMAP CEA/CNRS/ENSICAEN, Box 5133, F-14070 Caen cedex 5, France \\ ${ }^{4}$ Institut für Kernphysik, Universität Frankfurt, D-60486 Frankfurt, Germany \\ ${ }^{5}$ A. A. Noyes Laboratory of Chemical Physics, California Institute of Technology, Pasadena, California 91125, USA \\ ${ }^{6}$ Department of Material Science, Himeji Institute of Technology, Kamigori, Hyogo 678-1597, Japan \\ ${ }^{7}$ National Metrology Institute of Japan, AIST, Tsukuba 305-8568, Japan
}

(Received 19 December 2007; published 20 August 2008; corrected 26 August 2008)

\begin{abstract}
We report results of measurements and of Hartree-Fock level calculations of molecular-frame photoelectron angular distributions (MFPADs) for $\mathrm{C} 1 s$ photoemission from $\mathrm{CO}_{2}$. The agreement between the measured and calculated MFPADs is on average reasonable. The measured MFPADs display a weak but definite asymmetry with respect to the $\mathrm{O}^{+}$and $\mathrm{CO}^{+}$fragment ions at certain energies, providing evidence for an overlap of gerade and ungerade final ionic states giving rise to a partial breakdown of the two-step model of core-level photoionization and its subsequent Auger decay.
\end{abstract}

DOI: 10.1103/PhysRevLett.101.083001

PACS numbers: 33.60.+q, 33.80.- $\mathrm{b}, 34.80 .-\mathrm{i}$

Interference effects have been intriguing from the very beginning of quantum mechanics. Consider, for example, the processes of core-level photoionization and subsequent Auger decay. These processes are often considered not to be correlated and are thus described separately within a two-step model. However, quantum interference may lead to a breakdown of this well-known two-step model. Strictly speaking, the two-step model assumes there is an isolated intermediate state which is excited in the first step and then decays in the second step. An obvious consequence of the model is that the outcome of an Auger experiment can be predicted from a knowledge of the properties of a single intermediate state and of the transition amplitude from that state into the Auger final state. On this basis the two-step model should be violated if there is interference between quantum paths with different intermediate states, as discussed previously both for atoms [1] and molecules [2]. The overlap between different electronic states due to lifetime broadening plays a role in atoms, whereas vibrational motion in the intermediate state plays a major role in molecules.

An alternative view of the two-step model is that the outcome of the photoionization and Auger experiments can be predicted without accounting for the interaction between the photoelectron and the Auger electron or the postcollision interaction (PCI). PCI plays an essential role in this class of breakdown. From this point of view, two interesting and contradictory results have been reported for molecular-frame Auger electron angular distributions for $\mathrm{CO}$ molecules. Guillemin et al. observed a strong variation of the angular distribution with the initial ionization channel ( $\Sigma$ or $\Pi$ ) and with photon energy which they attributed to the breakdown of the two-step model [3]. Later, Weber et al. revisited this problem and reported results in disagreement with those of Guillemin et al., concluding that the two-step model holds [4].

These discussions of the breakdown of the two-step model focus on its influence on the Auger spectrum. Recently, Scheit and Cederbaum [5] noted that the photoelectron spectrum may also be influenced by the breakdown of the two-step model, if the spectrum is recorded in coincidence. Their emphasis was on the modification of the vibrational distribution in the photoelectron spectrum and the role of nuclear dynamics. In the present contribution, we discuss the photoelectron angular distributions recorded in coincidence with fragment ions as an indication of partial breakdown of the two-step model.

The most natural reference frame in which to consider molecular photoionization is the molecular frame. Experimentally, this can be realized by angle-resolved photoelectron-photoion coincidence measurements as demonstrated by Shigemasa et al. [6]. Position sensitive detectors have enabled a remarkable breakthrough in measurements of molecular-frame photoelectron angular distributions (MFPADs). To date, these multicoincidence momentum imaging techniques have been extensively employed to study the MFPADs of linear molecules [7-13]. This approach to MFPAD measurements, however, implicitly assumes the two-step model, in which the MFPAD is assumed to be factored out from the subsequent decay.

To discuss the breakdown of the two-step model in MFPAD measurements, we revisit the $\mathrm{C} K$-shell photoionization of $\mathrm{CO}_{2}$ and record $\mathrm{C} 1 s$ photoelectrons in coincidence with $\mathrm{O}^{+}$and $\mathrm{CO}^{+}$. An asymmetry in the MFPADs 
for photoemission from the central $\mathrm{C}$ atom relative to the $\mathrm{O}^{+}$and $\mathrm{CO}^{+}$directions would constitute evidence for the breakdown of the two-step model because the factorization within the two-step model would, in fact, restore the symmetry of the MFPAD for $1 s$ photoemission from the central $\mathrm{C}$ atom. Thus, the asymmetry, if observed, would directly indicate that the photoemission process is correlated with subsequent anisotropic processes and would imply a breakdown of the two-step model.

The experiment was carried out on the $c$ branch of the soft x-ray photochemistry beam line 27SU [14-16] at SPring- 8 and was practically the same as previously reported with the preliminary results [11]. Our coincidence momentum imaging apparatus was based on measurements of electron and ion time-of-flight (TOF) with two multihit two-dimensional position sensitive detectors which was equivalent to cold-target recoil momentum spectroscopy or reaction microscope [17]. A supersonic jet of $\mathrm{CO}_{2}$ in the vertical direction crossed the photon beam in the horizontal direction. The TOF axis was in the horizontal direction and perpendicular to both the gas and photon beams. Photoelectrons and fragment ions ejected over $4 \pi$ steradians were driven to each of the electron and ion detectors using uniform electrostatic and magnetic fields. Each detector was equipped with a 2-dimensional (2D) multihit readout delay-line anode (Roentdek), which permitted measurements of both the time of detection and the $2 \mathrm{D}$ position coordinates and thus allowed us to extract 3-dimensional (3D) momenta. We recorded only events in which at least two ions and one electron were detected in coincidence. The orientation of the molecular axis at the time of photoemission was extracted from the momentum vectors of the $\mathrm{CO}^{+}$and $\mathrm{O}^{+}$fragments resulting from Coulomb dissociation of $\mathrm{CO}_{2}^{2+}$ subsequent to rapid Auger decay. The measurements were performed at seven different photon energies: $303.8,307,311.8,312.2,319.4$, 320.9 , and $329.8 \mathrm{eV}$. Here, the C $1 s$ ionization energy is $297.63 \mathrm{eV}$ [18]. At four energies $(303.8,307,312.2$, and $320.9 \mathrm{eV}$ ) we recorded two sets of spectra using horizontal and vertical polarizations.

In the analysis of the experimental data, we have employed the projection method $[19,20]$. In this method, all possible experimental information is encapsulated in four one-dimensional (1D) functions. Since we use all the data points to obtain the four 1D functions, the statistical data analysis is improved significantly compared with the conventional approach where the reaction plane is predefined (see, e.g., [11,12]). A similarly important advantage is that data from all directions in the laboratory frame are used in the projection method which, to first order, averages out possible systematic errors. With the resulting four 1D functions, we can reconstruct MFPADs at each energy for any angle between the molecular axis and the $E$ vector [21].

We have also calculated photoelectron angular distributions within a relaxed-core Hartree-Fock (RCHF) approxi- mation using a molecular basis set obtained with Slater's transition-state approximation [22]. To obtain the photoelectron orbitals we employed an iterative procedure to solve the Lippmann-Schwinger equation associated with the one-electron Schrödinger equation [23] with a potential produced by the transition-state orbitals. The calculations are practically the same as reported previously [11].

To compare the measured and calculated MFPADs, we selected the reaction in which the electron is emitted in the plane defined by the molecular axis and the $E$ vector and reconstructed the MFPADs in this reaction plane, as shown in Fig. 1. Here the angle $\theta_{n}$ between the $E$ vector and

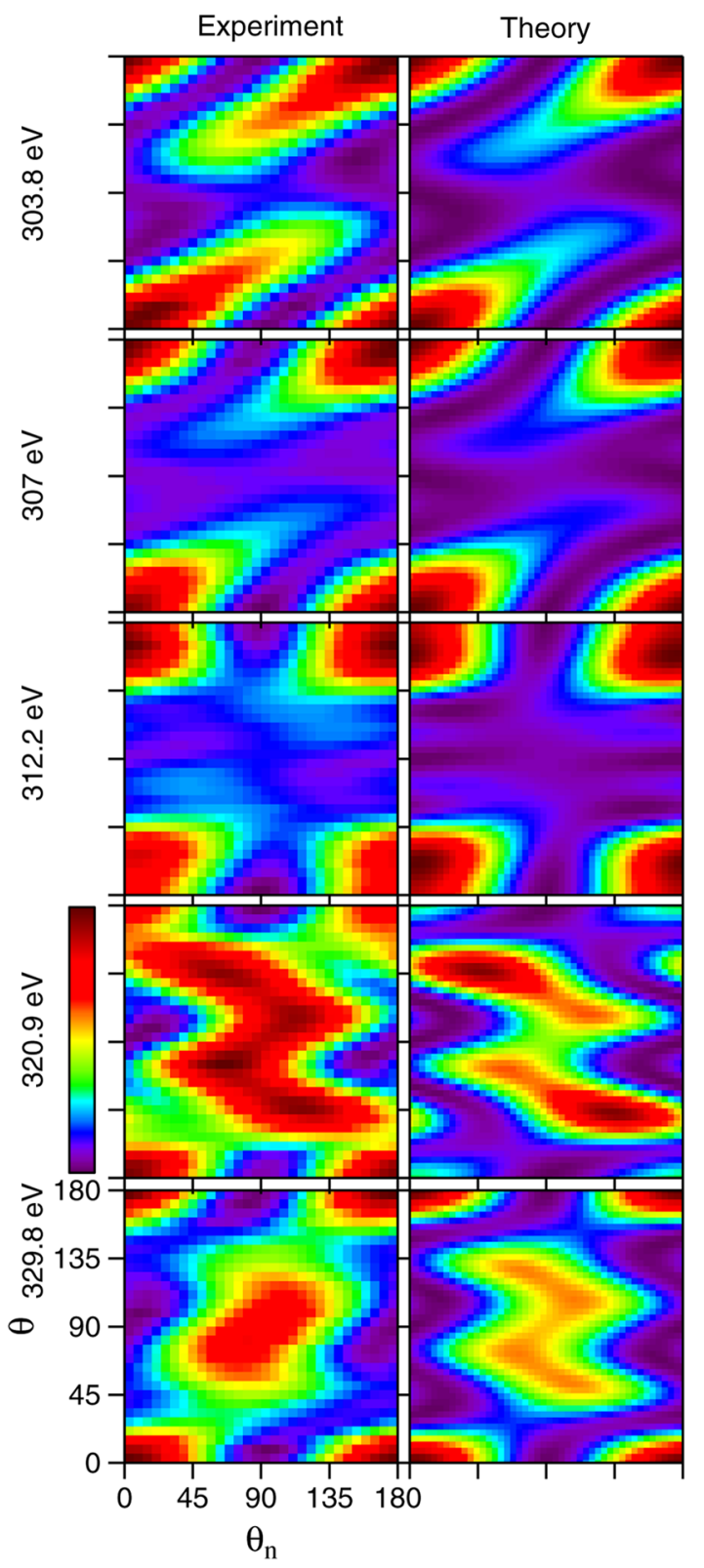

FIG. 1 (color online). Experimental and theoretical 2D plots of C $1 s$ MFPADs of $\mathrm{CO}_{2}$ at photon energies 303.8, 307, 312.2, 320.9 , and $329.8 \mathrm{eV} . \theta=0^{\circ}$ and $180^{\circ}$ are the directions of $\mathrm{O}^{+}$ and $\mathrm{CO}^{+}$, respectively. See text for details. 
molecular axis is shown along the horizontal axis and the electron emission polar angle $\theta$ relative to the molecular axis is shown along the vertical axis. The electron emission intensity is plotted in a gray scale (color scale online), at five photon energies.

At first glance, the agreement between experiment and theory is reasonable. In this figure, $\theta_{n}=0^{\circ}$ and $90^{\circ}$ correspond to $\Sigma \rightarrow \Sigma$ and $\Sigma \rightarrow \Pi$ transitions, respectively. The shape resonance is located at $312 \mathrm{eV}$, where the electron emission is mostly at $\theta_{n}=0^{\circ}(\Sigma \rightarrow \Sigma)$ and along the molecular axis. The calculated MFPADs are also seen to always exhibit symmetry relative to the center because of the assumed $D_{\infty h}$ molecular symmetry. However, we should note that the measured MFPADs do not show this complete point symmetry at 312.2 and $320.9 \mathrm{eV}$.

To confirm this asymmetry, in Fig. 2, we made polar plots of the electron emission at $\theta_{n}=0$ for six energies. Here, the polarization vector is parallel to the molecular axis. The solid curves represent the simulation. The distribution at each energy is normalized so that the integrated intensity (area enclosed) is the same for the measured and calculated spectra. In this plot the disagreement between the measured and calculated spectra at $\sim 320 \mathrm{eV}$, as discussed previously in Ref. [11], becomes apparent. Besides, we can clearly see that, though the calculated MFPADs are always symmetric, the measured MFPADs at 311.8 and $312.2 \mathrm{eV}$, as well as at 319.4 and $320.9 \mathrm{eV}$, exhibit asymmetry. Here, the MFPADs at 312.2 and $320.9 \mathrm{eV}$ were recorded with horizontal polarization, whereas the MFPADs at 311.8 and $319.4 \mathrm{eV}$ were recorded with vertical polarization with slightly different field conditions at a different beam time, for confirmation. We also investigated the dependence of the MFPADs on kinetic energy release (KER); the MFPADs are essentially the same as shown in Figs. 1 and 2, as long as the KER is larger than $4 \mathrm{eV}$. This confirms that the axial recoil approximation holds [4], and both Auger decay ( $\sim 7 \mathrm{fs})$ and dissociation ( $\sim 10 \mathrm{fs})$ can be assumed to be much faster than molecular rotations with its time scale of the order of ps. In our preliminary report [11], we could not identify this asymmetry because of the low statistics of the experimental data: significant improvement in the statistics of the data, afforded by the projection method, allowed us to pindown the asymmetry as evidence of partial breakdown of the two-step model.

We have previously observed a similar asymmetry in the $\mathrm{O} 1 s$ MFPADs [12]. Starting with delocalized O $1 s \sigma_{g}$ and $\sigma_{u}$ core holes, the observed asymmetry can be interpreted as due to interference between these two intermediate states and thus as a signature of the breakdown of the two-step model. The observations, however, are to be rationalized as follows. Antisymmetric stretching vibrations are known to arise in the $\mathrm{O} 1 s$ ionized state [24,25]. The $\mathrm{CO}^{2+}$ ion has an asymmetric equilibrium configuration with respect to the plane perpendicular to the molecular axis and dynamical core-hole localization is realized

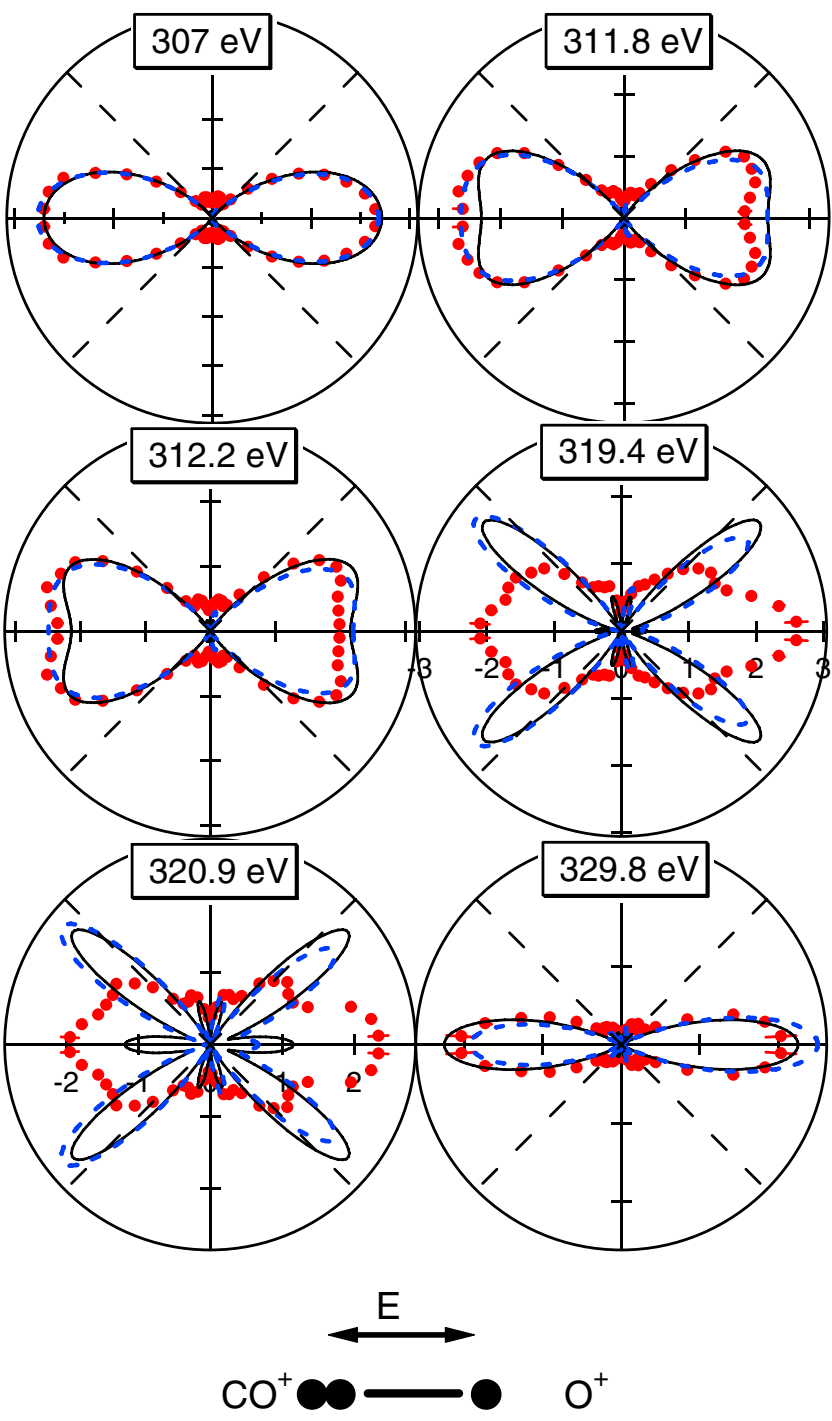

FIG. 2 (color online). Polar plots of $\mathrm{C} 1 s$ MFPADs of $\mathrm{CO}_{2}$ with molecular orientation parallel to the $E$ vector at photon energies 307, 311.8, 312.2, 319.4, 320.9, and $329.8 \mathrm{eV}$. The dots correspond to measurements. Solid and dashed curves are calculations with symmetric and asymmetric geometries, respectively. See text.

through vibronic coupling. As a result, diabatic representations of the symmetry-broken potential energy curves are more realistic than the adiabatic representations of symmetry-adapted potential energy curves. One can thus expect that the $\mathrm{C}-\mathrm{O}$ bond, with a core hole on the oxygen atom, is already slightly elongated at photoionization. In this case, the core hole resides on the $\mathrm{O}^{+}$ion and an ejected electron thus experiences an asymmetric potential resulting in reflection asymmetry in the MFPADs. In a sense, asymmetry in the O $1 s$ MFPADs due to dynamical corehole localization is to be expected.

The observed asymmetry of the C $1 s$ MFPADs cannot be explained by the same line of argument as for the $\mathrm{O} 1 \mathrm{~s}$ MFPADs. How does coincident detection of photoelec- 
trons with asymmetric fragmentation $\mathrm{O}^{+}-\mathrm{CO}^{+}$affect the correlated photoelectron angular distributions? One obvious possibility would be that we are probing MFPADs from an asymmetric geometry by detecting asymmetric fragmentation. We thus carried out calculations of the MFPADs for several displacements of the $\mathrm{C}$ and $\mathrm{O}$ atoms with a harmonic potential for the ground asymmetrical vibrational mode. In Fig. 2, the dashed lines show the results with the $\mathrm{C}$ and $\mathrm{O}$ atoms at their root mean square displacements in this potential (CO distances of 2.3147 and 2.0773 a.u.). As can be seen, the MFPADs calculated with this displacement certainly indicate an asymmetry and are in better agreement with the measurements at 311.8 and $312.2 \mathrm{eV}$. The MFPADs are rather insensitive to the displacement at the lower energies of $303.8 \mathrm{eV}$ (not shown here) and $307 \mathrm{eV}$. At energies of 319.4 and $320.9 \mathrm{eV}$, the calculations predict a much richer structure than observed, as noted previously [11], and one cannot judge whether this displacement results in better agreement with the observations. At $329.8 \mathrm{eV}$, the same displacement predicts significant asymmetry in the MFPAD, while the observed MFPAD is symmetric. These findings imply that the observed asymmetry of the MFPADs may be related to the asymmetric geometry but also depend on the specific photon energies, indicating the effect of the resonance.

We note that similar asymmetric molecular-frame angular distributions have been observed in valence photoemission of homonuclear diatomic molecules [26-28]. In these cases, close-lying gerade and ungerade symmetry states give rise to interference terms. In the present work, we offer the first evidence of asymmetric MFPADs from a symmetric molecule for core-level photoemission from the central atom, which subsequently undergoes Auger decay. Here, as in valence photoionization, the interference term between the gerade and ungerade symmetry states is considered the cause of the asymmetric MFPADs, representing a partial breakdown of the two-step model. A recent study on the photoelectron satellite observed at the shape resonance suggested that $2 \sigma_{g}^{-1} 4 \sigma_{u}$ shape resonance couples to conjugate satellite continuum state $2 \sigma_{g}^{-1} 1 \pi_{g}^{-1} 2 \pi_{u}^{1} \epsilon \sigma_{g}$, resonantly enhancing the latter satellite cross section [29]. We speculate that this coupling may generate the interference term, resulting in partial breakdown of the two-step model. The results at $\sim 320 \mathrm{eV}$ may also be interpreted as a consequence of similar coupling between the $2 \sigma_{g}^{-1} \epsilon \sigma_{u}$ continuum state and the conjugate satellite continuum state, $\mathrm{S} 3$ designated by Schmidbauer et al. [30], which appears at $\sim 320 \mathrm{eV}$. Our hypothesis here assumes that an interaction between the photoelectron and the valence electron causes the interference responsible for the partial breakdown of the two-step model and that PCI plays no role.

In summary, we have reported on the results of measurements and calculations of MFPADs for $\mathrm{C} 1 s$ photoemission from $\mathrm{CO}_{2}$. Contrary to the calculated MFPADs, the measured MFPADs display a weak but definite asymmetry with respect to the central $\mathrm{C}$ atom, providing evidence for an overlap of the gerade and ungerade symmetry states of the molecular ion resulting in their coherent superposition and consequently a partial breakdown of the two-step model of the Auger decay.

This experiment was carried out with the approval of JASRI and supported in part by Grants-in-Aid for Scientific Research from the Japan Society for the Promotion of Science (JSPS). The authors are grateful to L. S. Cederbaum, U. Hergenhahn, and N. M. Kabachnik for stimulating discussions and to the staff of SPring-8 for their technical assistance. X. J. L. and A. D. acknowledge financial support from J.S.P.S. and R.D., L.S., and A.C. acknowledge support by DFG.

*ueda@tagen.tohoku.ac.jp

[1] N. M. Kabachnik et al., Phys. Rep. 451, 155 (2007).

[2] R. Püttner et al., Phys. Rev. A 59, 4438 (1999).

[3] R. Guillemin et al., Phys. Rev. Lett. 87, 203001 (2001).

[4] Th. Weber et al., Phys. Rev. Lett. 90, 153003 (2003).

[5] S. Scheit and L.S. Cederbaum, Phys. Rev. Lett. 96, 233001 (2006).

[6] E. Shigemasa et al., Phys. Rev. Lett. 74, 359 (1995).

[7] F. Heiser et al., Phys. Rev. Lett. 79, 2435 (1997).

[8] A. Lafosse et al., Phys. Rev. Lett. 84, 5987 (2000).

[9] M. Takahashi, J. P. Cave, and J.H. D. Eland, Rev. Sci. Instrum. 71, 1337 (2000).

[10] Th. Weber et al., J. Phys. B 34, 3669 (2001).

[11] N. Saito et al., J. Phys. B 36, L25 (2003).

[12] N. Saito et al., J. Phys. B 38, L277 (2005).

[13] T. Teramoto et al., J. Phys. B 40, F241 (2007).

[14] H. Ohashi et al., Nucl. Instrum. Methods Phys. Res., Sect. A 467-468, 529 (2001).

[15] H. Ohashi et al., Nucl. Instrum. Methods Phys. Res., Sect. A 467-468, 533 (2001).

[16] K. Ueda, J. Phys. B 36, R1 (2003).

[17] J. Ullrich et al., Rep. Prog. Phys. 66, 1463 (2003).

[18] K. C. Prince et al., J. Phys. B 32, 2551 (1999).

[19] R.R. Lucchese et al., J. Electron Spectrosc. Relat. Phenom. 155, 95 (2007).

[20] X.-J. Liu et al., J. Phys. B 40, 485 (2007).

[21] R. R. Lucchese et al., Phys. Rev. A 65, 020702(R) (2002).

[22] J.C. Slater, The Self-Consistent Field for Molecules and Solids: Quantum Theory of Molecules and Solids (McGraw-Hill, New York, 1974), Vol. 4.

[23] R. R. Lucchese et al., Phys. Rev. A 25, 2572 (1982).

[24] W. Domcke and L. S. Cederbaum, Chem. Phys. 25, 189 (1977).

[25] A. Kivimäki et al., Phys. Rev. Lett. 79, 998 (1997).

[26] A. V. Golovin et al., Phys. Rev. Lett. 79, 4554 (1997).

[27] A. Lafosse et al., J. Phys. B 36, 4683 (2003).

[28] F. Martin et al., Science 315, 629 (2007).

[29] X.-J. Liu et al., Phys. Rev. Lett. 101, 023001 (2008).

[30] M. Schmidbauer et al., Phys. Rev. A 52, 2095 (1995). 